\title{
COMMISSION 35: STELLAR CONSTITUTION
}

\section{(CONSTITUTION DES ETOILES)}

\section{PRESIDENT: J.-P. Zahn}

VICE-PRESIDENT: D. VandenBerg

ORGANIZING COMMITTEE: R. Canal, C. Chiosi, W. Dziembowski, J. Guzik, G. Meynet, G. Michaud, A. Renzini, H. Saio, A. Tutukov \& G. Vauclair

\section{INTRODUCTION}

Our Commission decided to proceed as before, with a rather comprehensive report, while focusing on the subjects where most progress has been achieved during the past three years. The colleagues who kindly contributed to it are W. Dziembowski (helio- and asteroseismology), J. Guzik (intermediate-mass stars), G. Meynet (massive stars), G. Michaud (atomic diffusion), D. VandenBerg (low mass stars), G. Vauclair (white dwarfs), J.-P. Zahn (convection, rotational mixing).

\section{WORKSHOPS, COLLOQUIA AND CONFERENCES}

\subsection{Of direct interest to Commission 35}

New Eyes to See Inside the Sun and Stars, IAU Symp. 185, Kyoto, Japan, 1997 [ed. F.-L. Deubner, J. Christensen-Dalsgaard \& D. Kurtz; Kluwer]

Cosmic Chemical Evolution, IAU Symp. 187, Kyoto, 1997 [K. Nomoto \& J.W. Truran; Kluwer]

Abundance Ratios in the Oldest Stars, Joint Discussion 1, Kyoto, $1997^{1}$

Stellar Evolution in Real Time, Joint Discussion 8, Kyoto, $1997^{1}$

Low Luminosity Stars, Joint Discussion 10, Kyoto, $1997^{1}$

The First Results of Hipparcos and Tycho, Joint Discussion 14, Kyoto, $1997^{1}$

ISO's View on Stellar Evolution, Noordwijkerhout, Netherland, 1997

Cool Stars, Stellar Systems, and the Sun, Cambridge, USA, 1997 [ed. Donahue \& J.A. Bookbinder; ASP Conf. Ser. 154]

ITP Conference on Supernova Explosions: their Causes and Consequences, Santa Barbara, USA, 1997

Stellar Astrophysics, Pacific Ring Conference, Hong Kong, 1997 [ed. K.L. Chan, K.S. Cheng \& H. P. Singh; ASP Conf. Ser. 138]

Herbig-Haro Flows and the Birth of Low Mass Stars, IAU Symp. 182, Chamonix, France, 1997 [ed. B. Reipurth \& C. Bertout; Kluwer]

Asymptotic Giant Branch Stars, IAU Symp. 191, Montpellier, France, 1998 [ed. Th. Le Bertre, A. Lèbre, \& C. Waelkens; to appear in ASP Conf. Ser.]

Wolf-Rayet Phenomena in Massive Stars and Starburst Galaxies, IAU Symp. 193, Puerto Vallarta, Mexico, 1998 [ed. K.A. van der Hucht, G. Koenigsberger \& P.R.J. Eenens; to appear in ASP Conf. Ser.]

\footnotetext{
1 in Highlights of Astronomy, vol. 11 [ed. J. Andersen; Kluwer]
} 
Structure and Dynamics of the Interior of the Sun, SOHO6/GONG'98 workshop, Boston, USA, 1998 [ed. A. Wilson; ESA SP-418]

Solar Composition and its Evolution - from Core to Corona, ISSI workshop Berne, Switzerland, 1998 [ed. C. Fröhlich, M.C.E. Huber, S.K. Solanki \& R. Von Steiger; Kluwer]

Theory and Tests of Convection in Stellar Structure, Granada, Spain, 1998 [ed. A. Gimenez \& B. Montesimos; ASP Conf. Ser. 173]

The Light Elements and Their Evolution, IAU Symposium 198, Natal, Brazil, 1999 [ed. L. da Silva, R. de Medeiros \& M. Spite]

Cool Stars, Stellar Systems, and the Sun, Tenerife, Spain, 1999 [ed. R.J. Garcia Lopez, R. Rebolo \& M.R. Zapaterio Osorio; to appear in ASP Conf. Ser.]

Astrophysical Turbulence and Convection, 14th Florida workshop, Gainesville, USA, 1999 [ed. J.R. Buchler \& H. Kandrup; to appear in Annals New York Acad. Sci.]

Helioseismology at Intermediate and High-Degree, GONG'99 Workshop, Tucson, USA, 1999 [abstracts available at http://www.gong.noao.edu/papers/gong.99/]

\subsection{Other meetings of interest}

Fundamental Stellar Properties: the Interaction between Observations and Theory, IAU Symp. 189, Sydney, Australia, 1997 [ed. T.R. Bedding, A.J. Booth \& J. Davies; Kluwer] A Half Century of Stellar Pulsation Interpretations, Los Alamos, USA, 1997 [ed. P.A. Bradley \& J.A. Guzik; ASP Conf. Ser. 135]

Fourth Whole Earth Telescope Workshop, Koninki, Poland, 1997, [ed. E.G. Meistas \& P. Moskalik; Baltic Astronomy 7, Nos. 1-2, 1998]

New Views of the Magellanic Clouds, IAU Symp. 190, Victoria, Canada,1998 [ed. Y.H. Chu, N. Suntzeff, J. Hesser \& D. Bohlender; Kluwer]

Variable and Non-spherical Stellar Winds, IAU Colloq. 169, Heidelberg, Germany, 1998 [eds. B. Wolf, A.W. Fullerton \& O. Stahl; Lecture Notes in Phys. 532, Springer]

Precise Stellar Radial Velocities, IAU Colloq. 170, Victoria, Canada, 1998 [ed. C.D. Scarfe \& J.B. Hearnshaw; in preparation]

Astrophysical Fluids - From Atomic Nuclei to Stars and Galaxies, Haifa, Israel, 1998 [ed. D. Prialnik \& O. Regev; Phys. Reports vol. 311, \# 3-5]

6th Vienna Workshop in Astrophysics on delta Scuti and Related Stars, Vienna, 1999 [ed. M. Breger \& M. Montgomery; to appear in ASP Conf. Ser.]

Variable Stars as Important Astrophysical Tools, NATO ASI, Cesme, Turkey, 1998, [ed. C. Ibanoglu; Kluwer]

Eta Carinae at the Millenium, Gallatin Gateway, USA, 1998 [ed. J. Morse, R. Humphreys \& A. Damineli; ASP Conf. Ser. 179]

The Impact of Large-Scale Surveys on Pulsating Star Research, IAU Colloq. 176, Budapest, Hungary, 1999, [ed. L. Szabados \& D. Kurtz; to appear in ASP Conf. Ser.]

Stellar Clusters and Associations: Convection, Rotation, and Dynamos, Palermo, Italy, 1999 [ed. R. Pallavicini, G. Micela \& S. Sciortino; to appear in ASP Conf. Ser.]

Spectrophotometric Dating of Stars and Galaxies, Annapolis, USA, 1999 [ed. I. Hubeny, S. Heap \& R. Cornett; to appear in ASP Conf. Ser.]

\section{MASSIVE STARS}

\subsection{Massive star formation and evolution}

Massive stars are wonderful objects to probe the process of star formation and evolution in environments differing by the metallicity (e.g. Massey \& Johnson 1998, ApJ 505, 793), the star formation rate (e.g. Gonzalez Delgado et al. 1999, ApJ 513, 707), the redshift (Steidel 
et al. 1999, ApJ 519, 1) etc... In particular, observations of low metallicity regions provide important templates for studying star formation in the very distant metal-poor galaxies which populate the early universe. New facilities, as for instance the NICMOS/HST or the ISO satellites, have enabled to detect and study, sometimes for the first time, individual massive stars in very crowded and obscured regions, as for instance at the Galactic center (Serabyn et al. 1998, Nature 394, 448; Figer et al. 1999; ApJ 514, 202), in compact HII regions (Heydari et al. 1999, A\&A 344, 848) or in the cluster R136a of 30 Doradus (e.g. Crowther \& Dessart 1998, MNRAS 296, 622; de Koter et al. 1998, ApJ 509, 879).

The formation process of massive stars remains largely unknown. Our ignorance comes essentially from the fact that this phase is quite difficult to observe being obscured by dense clouds of gas and dust. This leaves open various theoretical models for massive star formation: are they formed by accretion-induced collisions of intermediate-mass stars in the cores of dense stellar clusters (Bonnell et al. 1998, MNRAS 298, 93) or do they form by mass accretion as their lower mass counterparts (Maeder 1998, IAU Symp. 192, 20). On these issues depend important physical quantities as the upper mass limit formed and its (possible) dependence on the metallicity, the distribution of the initial rotational velocities, the spatial distribution of the massive stars in young clusters, the dependence of the IMF on the star formation efficiency, the evolution of very young starbursts.

Different groups are currently producing massive star models, at Padova (cf. Salasnich et al. 1999, A\&A 342, 131), at Geneva (cf. Mowlavi et al. 1998, A\&AS 128, 471), Langer et al. (e.g. 1999, ApJ 520, 49), Stothers \& Chin (e.g. 1999, ApJ 522, 960), Claret \& Gimenez (e.g. 1998, A\&AS 133, 123), Chieffi et al. (1998, ApJ 502, 737). Evolutionary models of massive stars in two dimensions have recently been computed exploring the effects of convection (Bazan \& Arnett, 1998, ApJ 496, 316) and of rotation (Deupree, 1998, ApJ 499, 340 ). With respect to one dimensional computations, these works show new phenomena which may have important consequences for our understanding of supernova explosion and nucleosynthesis.

Many observational features point towards the need for an extra-mixing process in massive star models. As an example all fast rotators among the $\mathrm{O}$-stars present $\mathrm{He}$-excesses at their surface (e.g. Herrero et al. 1999, A\&A 348, 542). Using the recent advances made in our understanding of the physical effects induced by rotation (Maeder 1997 A\&A 321,134; Talon \& Zahn 1997, A\&A 317, 749; Maeder \& Zahn 1998, A\&A 334, 1000), some groups have explored the effects of rotation on massive star evolution (Talon et al. 1997, A\&A 322, 209; Meynet \& Maeder 1997, A\&A 321, 465; Heger \& Langer 1998, A\&A 324, 210; Denissenkov et al. 1999, A\&A 341, 181). The results are very promising and have important consequences for nucleosynthesis (Heger et al. 1999, ApJ in press) and population synthesis models. Moreover rotation may also shape the circumstellar environment around massive stars (Maeder 1999; A\&A 347, 185; Langer et al. 1999, ApJ 520, L49).

\subsection{Luminous Blue Variables and Wolf-Rayet stars}

The evolutionary scenarios leading to the formation of these stars remain a quite debated question (e.g. Stothers \& Chin 1999, ApJ 522, 960). The chemical composition of the nebulae surrounding the Luminous Blue Variables (LBV) stars and some Wolf-Rayet (WR) stars, their kinematic properties and masses put very important constraints on the past evolution of these stars (cf. Smith et al. 1998, ApJ 503, 278). For example, R143 (a LBV in the LMC), has a nebula which presents abundance pattern similar to that determined for the inner ring of SN $1987 \mathrm{~A}$, which is thought to be composed of red supergiant (RSG) wind material. The observed low expansion velocity also suggests that the nebula was once the CN-processed convective envelope of a RSG. In contrast $\eta$ Car has an ejected nebula composed of heavily processed CNO material (e.g. Dufour et al. 1997, ASP Conf. Ser. 120, 255) and thus results likely from the ejection by a W-R star.

W-R stars are the most advanced observed stage of massive star evolution and provide unique nucleosynthesis test. The fourth catalogue of Population I Wolf-Rayet stars in the Large Magellanic Cloud contains data on 134 W-R stars (Breysacher et al. 1999, A\&AS 
137, 117). The VIIth catalogue of galactic population I Wolf-Rayet stars (van der Hucht 1999, New Astronomy Reviews, to be submitted; see also IAU Symp. 193, p. 13) provide data for 218 galactic W-R stars (117 WN, $87 \mathrm{WC}, 11 \mathrm{WN} / \mathrm{WC}, 3 \mathrm{WO}$ ). Thanks to ISO observations, the large predicted overabundance of ${ }^{22} \mathrm{Ne}$ at the surface of the WC stars has been confirmed (Willis et al. 1997, MNRAS 290, 371). Since this element is one of the main neutron sources for the s-process in massive stars, this observation sets on firmer grounds the theoretical studies of this nucleosynthetic process. Moreover it gives also support to the hypothesis that the high isotopic ratio of ${ }^{22} \mathrm{Ne} /{ }^{20} \mathrm{Ne}$ observed in the galactic cosmic sources (Webber et al. 1997, ApJ 476, 766) may be due to the injection of WC material.

In the recent years many observations have accumulated pointing towards a clumped structure of the winds of Wolf-Rayet stars (Willis 1999, IAU Symp. 193, 1 and references therein). The neglect of clumping implies in general an overestimate of the mass loss rates deduced from spectroscopic analyses. A first attempt at re-deriving mass loss rates for WR stars from their radio Data accounting for the clumped structure and (potential) variable ionization in their outer winds has been given by Nugis et al. (1998, A\&A 333, 956). They find that the clumping-corrected mass loss rates are lower by a mean factor 1.5 for WN stars and 4 for WC stars compared to the values obtained by smooth wind models. Substantially lower mass-loss rates of W-R stars has importance in evolutionary model calculations and in reducing the problem of driving the W-R winds.

Thanks to their strong emission lines, Wolf-Rayet can be detected in the integrated spectrum of distant galaxies (see the new catalogue of Wolf-Rayet galaxies and highexcitation extra-galactic HII regions by Schaerer et al. 1999, A\&A 345, 17). The detection by Heckman et al. (1997, ApJ 482, 114) of a broad emission complex around the He II $\lambda 4686$ line that may be produced by an ensemble of about 30,000 Wolf-Rayet stars (WN subtype) in the type II Seyfert galaxy Mrk $477(=\mathrm{I} \mathrm{Zw} 92)$ is particularly interesting in the frame of a possible connection between AGN and starburst (e.g. Collin \& Zahn 1999, A\&A $344,433)$.

To investigate the regions of active star formation, Starburst99 (Leitherer et al. 1999, ApJS, 123, 3) is a very useful tool. This set of model predictions for spectrophotometric and related properties of galaxies with active star formation is available for retrieval at a Web site. The users can also run specific models with nonstandard parameters. The source code is available to the community.

\section{INTERMEDIATE-MASS STARS}

\subsection{Evolution}

Chiosi (1998, IAU Symp. 189, 323) reviews the evolution of intermediate-mass stars, with emphasis on treatments of convectively unstable regions and recent developments in AGB evolution theory. Ventura et al. (1998 A\&A 334, 953) present evolution calculations from the ZAMS to the RGB for 0.6 to $15 \mathrm{M}_{\odot}$ stars of solar-type composition. These calculations treat convection in the full spectrum of turbulence, considering billions of eddy length scales, with nuclear evolution fully coupled to turbulent transport. Ventura et al. use these results to rederive the ages of young clusters. Mowlavi et al. (1998 A\&AS 128, 471) present an evolutionary grid of 0.8 to $60 \mathrm{M}_{\odot}$ models of high $\mathrm{Y}, \mathrm{Z}(=0.48,0.1)$, including mass loss and moderate overshooting. This grid will be useful for studying high-metallicity stars in galactic bulges and elliptical galaxies. Deupree (1998, ApJ 499, 340) presents twodimensional evolution models including hydrodynamics for rotating $8.75 \mathrm{M}_{\odot}$ models. The hydrodynamics calculations show convective core overshooting equivalent to about 0.35 pressure scale heights on the ZAMS, and convective core rotation approximately constant on cylinders, decreasing with distance from the rotation axis. Siess, Forestini \& Bertout (1999, A\&A 342, 480) compute evolution models for accreting PMS stars of 0.1 to $2.5 \mathrm{M}_{\odot}$. They find that accretion accelerates evolution of the star as it moves down the Hayashi track, and affects surface abundances of deuterium and ${ }^{7} \mathrm{Li}$. 


\subsection{Nucleosynthesis and Yields}

A review of outstanding issues in the field of nucleosynthesis and yields from low and intermediate-mass stars, including stellar mixing processes, mass loss and ejection mechanisms, is given by Charbonnel (1998, ASP Conf. Ser. 147, 157).

Langer et al. (1999, A\&A 346, L37) present evolutionary models of intermediate-mass stars up to the thermally pulsing asymptotic giant branch (TP AGB) phase, including the effects of rotation on the stellar structure and rotational mixing of chemical species and angular momentum. They conclude that rotation is capable of producing a ${ }^{13} \mathrm{C}$ rich layer required for the s-process in TP AGB stars. Alternatively, Herwig et al. (1997, A\&A 324, L81) apply the results of a hydrodynamically based convective overshoot model of Freytag et al. (1996, A\&A 313, 497) to TP AGB model calculations, and find that their models develop a small pocket of ${ }^{13} \mathrm{C}$. Frost, Lattanzio \& Wood (1998, ApJ 500, 355) discover a new kind of thermal pulse in intermediate-mass AGB stars, in which deep dredge-up during normal thermal pulses leads to the formation of a long unburnt tail in the helium profile, which later ignites under partially degenerate conditions. In this kind of flash, about four times more carbon is dredged up than in a normal pulse.

Forestini \& Charbonnel (1998, A\&AS 123, 241) calculate abundances and isotopic ratios of light elements $\mathrm{Li}, \mathrm{C}, \mathrm{N}, \mathrm{O}, \mathrm{F}, \mathrm{Ne}, \mathrm{Mg}, \mathrm{Al}$, and $\mathrm{Si}$ during the TP AGB phase for 3 to $7 \mathrm{M}_{\odot}$ stars with $\mathrm{Z}=0.02$ and $\mathrm{Z}=0.005$. Denissenkov, Weiss \& Wagenhuber $(1997, A \& A$ 320,115 ) calculate light element nucleosynthesis for $\mathrm{Z}=0.0001$ intermediate-mass TP AGB stars. Mowlavi, Goriely \& Arnould (1998, A\&A 330, 206) address the survival of the pure s-process short-lived $\left(t_{1 / 2}=1.5 \times 10^{7}\right.$ y) radionuclide ${ }^{205} \mathrm{~Pb}$ in intermediate-mass AGB stars, important for cosmochemistry, and conclude that it is more likely to survive for stars of increasing mass or decreasing $\mathrm{Z}$.

Portinari, Chiosi \& Bressan (1998, A\&A 334, 505) present stellar yields of several elements for 6 to $120 \mathrm{M}_{\odot}$ stars based on the Padova evolution tracks, and apply these yields to the chemical evolution of the solar neighborhood. These complement the yields calculated using the Padova tracks by Marigo, Bressan \& Chiosi (1998, A\&A 331, 564; 1996, A\&A 313, 545 ) for 0.7 to $5 \mathrm{M}_{\odot}$ stars. Van Den Hoek \& Groenewegen (1997, A\&AS 123, 305 ) present theoretical yields, suitable for galactic chemical evolution models, for $\mathrm{H},{ }^{4} \mathrm{He}$, ${ }^{12} \mathrm{C},{ }^{13} \mathrm{C},{ }^{14} \mathrm{~N}$, and ${ }^{16} \mathrm{O}$ for 0.8 to $8 \mathrm{M}_{\odot}$ stars based on the Geneva group evolution tracks. Boothroyd \& Sackmann (1999, ApJ 510, 232) discuss "cool bottom processing" of CNO isotopes in RGB and AGB stars, and calculate the results of first and second dredge-up for $0.8-9 \mathrm{M}_{\odot}$ stars. They find that intermediate-mass stars are a prime source of ${ }^{13} \mathrm{C},{ }^{14} \mathrm{~N}$, and ${ }^{17} \mathrm{O}$.

Weiss, Wagenhuber \& Denissenkov, (1996, A\&A, 313, 581) confirm earlier results that stars up to $5 \mathrm{M}_{\odot}$ are net producers of ${ }^{3} \mathrm{He}$. However, including additional mixing beneath the convective envelope leads to carbon isotope anomalies observed in globular cluster red giants, but also to destruction of ${ }^{3} \mathrm{He}$. Mazzitelli, D'Antona \& Ventura (1999, A\&A 348, 846) present results for detailed computations of $\mathrm{Li}$ production by hot bottom burning in 3.5 to $6 \mathrm{M}_{\odot}$ AGB stars, discussing dependence on mass, metallicity, mass loss rate, and the treatment of convection.

\subsection{Herbig Ae/Be Stars}

Herbig $\mathrm{Ae} / \mathrm{Be}$ stars are pre-main sequence stars of 2 to $8 \mathrm{M}_{\odot}$. A major review of the properties of these stars is presented by Waters \& Waelkens (1998, ARA\&A 36, 233). Recent research has focused on addressing such questions as whether IR excesses indicate the existence of an accretion disk around these stars, what drives the observed stellar winds, and what is the source of strong $\mathrm{X}$-ray emission and chromospheric heating. The latter question is especially puzzling since, unlike their lower-mass counterparts, the T Tauri stars, these stars are not expected to have a subphotospheric convection zone that might contribute to producing magnetic fields or generating a chromosphere and corona. 
Infrared excesses found in Herbig $\mathrm{Ae} / \mathrm{Be}$ stars have been attributed to the presence of circumstellar disks. However, Polomski \& Telesco (1998, BAAS 193, 73.18) argue that companion stars and diffuse emission from dust can account for much of the IR excess, so that disks are not required. Mannings \& Sargent (1997, ApJ 490, 792) conclude that ordered velocity gradients around two Herbig $\mathrm{Ae} / \mathrm{Be}$ stars strongly suggest material orbiting in a disklike configuration. Miroshnichenko et al. (1999, ApJ 520, L115) conclude from radiative transfer calculations that the dust distribution has an extended spherical component that dominates the IR emission, and an embedded compact disk that dominates the millimeter and submillimeter emission.

Regarding the x-ray emission, Preibisch (1999, A\&A 345, 583) notes that a likely source of this emission, i.e. a corona produced by magnetic activity, is unexpected, again since these stars supposedly lack surface convection zones. For the object EC95, Preibisch proposes a short period of deuterium shell burning, which gives rise to convection near the stellar surface, a dynamo-induced B field, and a corona. However, Waters \& Waelkens (1998, ARA\&A 36, 233) argue that deuterium burning cannot generally explain Herbig $\mathrm{Ae} / \mathrm{Be} \mathrm{x}$-ray emission since some Herbig Ae/Be stars already on or near the main sequence that would have long ago finished $D$ burning still show such emission. Lignières, Catala \& Mangeney (1996 A\&A 314, 465L) propose a chromospheric heating mechanism whereby the breaking torque exerted by the wind generates turbulent motions below the surface which extract rotational energy to heat the chromosphere.

Corcoran \& Ray (1998, A\&A 331, 147) conclude from the similarities between spectroscopic observations of $\mathrm{T}$ Tauri and Herbig Ae/Be stars that Herbig $\mathrm{Ae} / \mathrm{Be}$ winds are driven by accretion from circumstellar disks. Strafella et al. (1998, ApJ 505, 299) examine Herbig $\mathrm{Ae} / \mathrm{Be}$ winds in light of growing evidence that these stars have a magnetic field and surface convection, and conclude that deposition of some of the convective energy in Alfvèn waves is required to explain the observed winds.

\subsection{Initial Mass Function}

Several groups have evaluated the initial mass function (IMF)for intermediate-mass stars in our Galaxy, as well as for star clusters in other galaxies, in order to assess the universality of the IMF, and possible dependence on metallicity or other cluster properties. Hunter et al. (1996, ApJ 468, 633) find that the slope of the IMF for 6 to $15 \mathrm{M}_{\odot}$ stars in the large OB association NGC 206 in M31 is $-1.4 \pm 0.05$, close to the Salpeter value of -1.35 . Hunter et al. $(1997$, ApJ 478,124$)$ find an IMF slope for 0.85 to $9 \mathrm{M}_{\odot}$ stars in the young LMC star cluster NGC 1818 of $-1.23 \pm 0.08$, also close to Salpeter. Hunter et al. conclude from their comparisons of many IMF slopes in clusters and associations in the local group, that the IMF slope is independent of spatial concentration, richness of the cluster, metallicity, and stellar mass range down to $0.85 \mathrm{M}_{\odot}$. However, Selman et al. $(1999$, A\&A 347, 532) find that the IMF of 2.8 to $120 \mathrm{M}_{\odot}$ stars in the central 15" of the starburst cluster NGC 2070 in 30 Doradus is flatter than Salpeter at the 2-3 sigma level. Elmgreen (1997, ApJ 486, 944) finds that theoretical models of the IMF using random sampling of mass in a turbulent fractal cloud, naturally produce a Salpeter power law at intermediate to high mass, and a flattening at low mass. Slightly shallower IMFs might be expected in regions with high levels of ionization and turbulence.

\subsection{Binaries}

Sandquist et al. (1998, ApJ 500, 909) perform 3D hydrodynamic simulations of the common envelope evolution of binary systems containing an AGB star of mass 3 or $5 \mathrm{M}_{\odot}$ with a main sequence star of mass 0.4 or $0.6 \mathrm{M}_{\odot}$. During the simulation, the orbital period decreases from about 200 days to about 1 day. They find that a third of the envelope gas becomes unbound, and about $40 \%$ of the gas is ejected by the end of the simulation. During the middle of the evolution, a differentially rotating thick disk surrounds the remnant binary briefly before energy input from the decaying orbit drives this mass away. 
Bennett, Brown \& Yang (1999, IAU Colloq. 170) describe the Precise Mass Project to determine the masses of main sequence A or B stars eclipsed by a G-M supergiant, in 20 selected binary systems. They will determine the radial velocity semi-amplitude of the late-type primary to within $25-50 \mathrm{~m} / \mathrm{sec}$. This data will be extremely useful to constrain theoretical evolution of intermediate-mass stars. A rigorous attempt by Iwamoto \& Saio (1999, ApJ 521, 297) to compare evolution models with three intermediate-mass binary systems V2291 Oph, $\alpha$ Aur, and $\eta$ And, with masses determined to within $7 \%$, proved somewhat disappointing, as each system required a different mixing length to pressure scale height ratio.

\subsection{Pulsating Stars}

Marconi \& Palla (1998, ApJ 507, L141) investigate linear and nonlinear pulsation properties of pre-main sequence models of 1 to $4 \mathrm{M}_{\odot}$ to derive the expected pulsation properties of Herbig Ae/Be stars located near the classical Cepheid $/ \delta$ Scuti instability strip. Pamyatnykh (1999, Acta Astron 49, 119) calculate the instability region for slowly pulsating B (SPB) stars, 3 to $6 \mathrm{M}_{\odot}$ nonradial gravity mode pulsators, and find that it coincides well with the observed domain of SPB stars. Kaye et al. (1999, PASP 111, 840) describe a new class of nonradial gravity mode pulsators of early-F spectral type, the $\gamma$ Doradus variables. Paunzen et al. (1998, A\&A 335, 583) discuss the pulsation properties of $\lambda$ Boo stars, metal-deficient main sequence A-F stars, and whether the observation that about half of these stars pulsate can be used to choose between accretion or diffusion as the cause for the metal deficiency. For additional detail on pulsating variables, see the IAU Commission 27 report.

\section{STARS OF LOW MASS}

Several large grids of evolutionary tracks, incorporating updated physics, were reported during the past three years. Cassisi et al. (1996, ApJ 459, 298; 1997, A\&A, 317, 108) provided extensive calculations for very low $Z$ 's $\left(10^{-5}\right.$ to $\left.10^{-10}\right)$. At extremely low metallicities, helium shell flashes appear to be especially strong, and the convection zone which is generated is able to reach the H-burning shell, causing the transfer of protons into the hot He-burning region and (possibly) the total or partial ejection of the stellar envelope. Straniero et al. (1997, ApJ 490,425) generated a valuable set of models for application to globular clusters: they show that, contrary to published claims (e.g., Castellani et al. 1997, A\&A, 322, 801), horizontal-branch (HB) luminosities are independent of whether helium and/or metal diffusion is taken into account. Although diffusion leads to reduced envelope helium contents in upper giant-branch (and HB) stars, it also delays the onset of the helium flash, resulting in increased core masses, which compensates for the lowered surface $Y$ values. As a result, predicted HB luminosities are essentially unchanged.

Grids of evolutionary tracks in the cases $Z=0.03$ and $Z=0.04$ were computed by Claret (1997, A\&AS 125, 439) and Bono et al. (1997, ApJ 189, 822), respectively: the latter give a particularly detailed description of the HB phases of super-metal-rich stars. The set of tracks for solar abundances produced by Ventura et al. (1998, A\&A, 334, 953) is notable for adopting the "Full Spectrum of Turbulence" model of convection, while the very large grid reported by Pols et al. (1998, MNRAS 298, 525) assumed an amount of convective core overshooting that was carefully constrained by empirical data for $\zeta$ Aurigae eclipsing binaries (Schröder et al. 1997, MNRAS 285, 696) and double-lined eclipsing binaries (Tout et al. 1997, MNRAS 289, 689). (Indeed, most of the grids mentioned above, which is not a complete list, make some allowance for core overshooting, when appropriate, given the now overwhelming evidence in favor of its existence. Further recent examples of the success of overshooting models to explain the C-M diagrams of older open clusters include the studies of NGC 6819 and NGC 7789 by Rosvick \& VandenBerg 1998, AJ 115, 1516 and Gim et al. 1998, PASP, 110, 1318, respectively.) 


\subsection{Pre-Main-Sequence Evolution}

The widely accepted accretion model for the pre-main-sequence (PMS) phase of lower mass stars (Stahler 1988, ApJ 332, 864) has been the subject of a number of interesting studies. Siess et al. (1997, A\& A, 326, 1001) investigated the consequences of different mass accretion rates, showing that the accretion process accelerates the evolution of a star. That is, the estimated age of a PMS star from its position on the H-R diagram is reduced by a factor of 2-3 compared with the predictions of models that neglect accretion. In a follow-up investigation, Siess et al. (1999, A\&A, 342, 480) argued that deuterium must be present at the surfaces of accreting intermediate-mass stars: they also evaluated the ramifications of accretion for the location and morphology of PMS tracks. An investigation of the pulsational properties of PMS stars was carried out by Marconi \& Palla (1998, ApJ 507, L141), who determined the location of the instability strip for such stars. In addition, Mendes et al. (1999, A\&A, 341, 174) studied the effects of rotation on lithium depletion during the PMS phase. They found that rotation tends to decrease the $\mathrm{Li}$ abundance when the star is fully convective, but to increase it when a radiative core develops.

\subsection{Horizontal Branch Stars}

Bono et al. (1997, A\&A, 121, 327; 1997, A\&A, 317, 171; 1997, ApJ 479, 279; 1997, ApJ 483, 811 ; 1997, ApJ 489, 822) have carried out extensive work on the pulsational properties of RR Lyrae stars over a broad range in mass and chemical composition. They use non-linear, non-local, time-dependent treatments of convection and, among other things, they derive the locations on the H-R diagram of the red and blue boundaries for pulsational instability and predict useful relations between period, mass, luminosity, and effective temperature. Bono et al. (1997, ApJ 477, 346) have also shown, for the first time, that it is possible for some $\delta$ Scuti stars to be stable second-overtone pulsators.

From an analysis of the dependence of $M_{V}$ on directly observable quantities in $\mathrm{RR}_{\mathrm{ab}}$ stars - including the period and the usual Fourier decomposition parameters $A_{1}$ and $\phi_{31}-$ Kovács \& Jurcsik $\left(1996\right.$, ApJ 466, L17) have argued that values of $d M_{V} / d[\mathrm{Fe} / \mathrm{H}]>0.19$ are precluded for variables in the Galactic globular clusters. A similar result, based on the locations of cluster variables in the $\log$ (period)- $M_{V}$ diagram, was obtained by Caputo (1997, MNRAS 284, 994). On a discouraging note, Kovács \& Kanbur (1998, MNRA 295, 834) have found that most hydrodynamical models of RR Lyraes do not reproduce empirical relations between the shapes of light curves and physical parameters well enough to enable one to determine accurate estimates of the physical parameters of stars from theoretical light and velocity curves.

Yi et al. (1997, ApJ 482,677) constructed new HB tracks for a wide range in metallicity $(0.0001 \leq Z \leq 0.1)$, and carried out a detailed analysis of the so-called "slow blue phase" (SBP) in the on-going quest to understand the uv-upturn phenomenon in giant elliptical galaxies. They show that metal-rich stars spend much more time in this $u v$-bright phase than those of lower $Z$ because of their lower mass and greater lifetimes. Only at high helium abundances $(Y \geq 0.4)$ is the SBP phase expected to have a long duration.

\subsection{Globular Cluster Stars}

The failure of canonical stellar models to explain the observed surface abundances in globular cluster (GC) stars continues to motivate a great deal of research to try to resolve this dilemma. Sweigart (1997, ApJ 474, L23) noted that a likely side effect of the mixing that causes the $\mathrm{CNO}, \mathrm{Na}, \mathrm{Al}$, and $\mathrm{Mg}$ abundance variations along the upper giant branches of many clusters is the dredge-up of helium from the vicinity of the $\mathrm{H}$-burning shell into the envelope. The He-mixed models were predicted to have increased RGB tip luminosities, implying enhanced mass loss rates and leading to brighter and bluer horizontal branches. This could account for the observed low gravities in these stars, alleviate the longstanding period-shift problem, and explain part of the second-parameter phenomenon. Denissenkov 
et al. $(1997, A \& A, 320,115)$ ruled out the possibility that contamination of the gas by an early generation of AGB stars is responsible for the observed $\mathrm{O}$ and $\mathrm{Al}$ abundance variations, and in a later study $(1998, A \& A, 333,926)$, they argued that there must be an undetected resonance in the ${ }^{24} \mathrm{Mg}(\mathrm{p}, \gamma)^{27} \mathrm{Al}$ reaction as well as episodic increases in the temperature of the H-burning shell up to $74 \times 10^{6} \mathrm{~K}$ if deep mixing is to explain the abundance data. The same conclusion concerning the ${ }^{24} \mathrm{Mg}$ proton capture rate was reached independently by Cavallo et al. (1998, ApJ 492, 575), who also showed that lower metallicity giants should experience greater variations in their surface chemistry, if deep mixing occurs, because they have hotter and wider H-burning shells.

Using the predictions of stellar models together with observed ${ }^{12} \mathrm{C} /{ }^{13} \mathrm{C}$ ratios, Charbonnel et al. (1998, A\&A, 332, 204) found that the inferred $\mu$-gradient, needed to shield the nuclear-burning regions of a star from extra mixing, agrees very well with that expected to stabilize meridional circulation - giving considerable support to the notion that the deep mixing which occurs in GCs is driven by stellar rotation. More recently, Sackmann \& Boothroyd (1999, ApJ 510, 217) found that, if deep mixing is assumed to reach to the same distance in temperature from the H-burning shell and that such mixing commences when the shell contacts the $\mu$-gradient established by the first dredge-up, then current observations of the ${ }^{12} \mathrm{C} /{ }^{13} \mathrm{C}$ ratio (including its variations with mass) of Population I and field Population II stars could be reproduced theoretically. However, they suggest that much more extensive mixing/processing is apparently needed to explain the surface chemistry of GC stars. Importantly, Fujimoto et al. (1999, ApJ 519, 733) have found that, if (by some as yet unknown mechanism) hydrogen were mixed into the helium core, then a hydrogen shell flash would be ignited which would cause the formation of a convective shell able to access H-rich layers and reinforce the flash. As the flash temperatures are considerably higher than those in the H-burning shell during quiescent evolution, processing of ${ }^{24} \mathrm{Mg}$ can occur to the extent needed to explain the observations in metal-poor GCs if deep mixing carries that material to the surface layers. Fujimoto et al. predict that changes in the surface $\mathrm{Mg}$ abundance should not occur in metal-rich clusters because the duration of the flash is too short at high metallicities. Moreover, they argue that enhanced surface helium abundances is an unavoidable consequence of the processing and dredge-up of the products of the $\mathrm{Mg}-\mathrm{Al}$ cycle.

Interesting work on blue stragglers in GCs has been carried out by Sills et al. (1997, ApJ 487, 290; 1999, ApJ 513, 428) and Ouellette \& Pritchet (1999, AJ 115, 2539). Both groups used standard stellar evolution codes to investigate the structure and evolution of stars that merge as the result of collisions: initial models were taken from smoothed particle hydrodynamics (SPH) simulations. One of the main results of those calculations is that, to satisfy the constraints provided by their observed distributions on the C-M plane, the collision products must not undergo significant amounts of mixing (either by convection or circulation currents) after the collision occurs. It is noted that grazing collisions should cause rapid rotation, but this is not seen, suggesting that angular momentum is transferred to a disk or companion star in such instances.

Despite the wealth of data provided by Hipparcos and the Hubble Space Telescope, precise (and accurate) estimates of both the absolute and relative ages of GCs have remained elusive. D'Antona et al. (1997, ApJ 477, 519) argued (prior to the release of Hipparcos parallaxes) that the most metal-deficient GCs were no older than $\sim 12$ Gyr. The 23 Gyr reduction from canonical estimates was caused, in part, by their adoption of the Canuto \& Mazzitelli (1991, ApJ 370, 295) theory of convection, which has the effect of of reducing the age at a fixed turnoff luminosity, and partly by their adoption of new horizontal-branch models (Caloi et al. 1997, A\&A, 320, 823), which imply increased cluster distances. Subsequent GC main-sequence fits to local subdwarfs having Hipparcos-based distances yielded ages ranging from 10-12 Gyr (Reid 1997, AJ 114, 161; Gratton et al. 1997, ApJ 491, 749; also see Chaboyer et al. 1998, ApJ 494, 96) to 13-14 Gyr (Pont et al. 1998, A\&A, 329, 87). Although many others have argued in support of ages near $11 \mathrm{Gyr}$ (see, e.g., Salaris et al. 1997, ApJ 479, 665; Cassisi et al. 1998, A\&AS 129, 267), most direct estimates of RR Lyrae luminosities - from Baade-Wesselink analyses (Fernley et al. 
1998, A\&A, 330, 515), statistical parallax studies (Gould \& Popowski 1998, ApJ 508, 844), and Hipparcos parallaxes (Gratton 1998, MNRAS 296, 739) - imply short distances and high ages. Thus $\mathrm{a} \pm 3 \mathrm{Gyr}$ uncertainty persists on the absolute $\mathrm{GC}$ ages.

As far as relative GC ages are concerned, the main development has been the report of new, extensive, very homogeneous photometry for 30 clusters by Saviane et al. (1999, in the Third Stromlo Symposium, ASP Conf. Ser. 165, 308), which indicates that the dispersion in age at a given $[\mathrm{Fe} / \mathrm{H}]$ is small (less than $\sim 1 \mathrm{Gyr}$ ) for the bulk of the sample and that there is little or no dependence of age on metallicity or Galactocentric distance. Very similar results were obtained by VandenBerg (1999, same proceedings, p. 46), though he finds an $\approx 2$ Gyr decrease in mean age as the metallicity increases from $[\mathrm{Fe} / \mathrm{H}] \sim-2.3$ to $\sim-1.3$.

\subsection{Stars of Very Low Mass}

One of the main themes of recent work on very low mass stars is the importance of using the best available non-grey model atmospheres as boundary conditions for the stellar interior models (Chabrier \& Baraffe 1997, A\&A, 327, 1039; Kroupa \& Tout 1997, MNRAS 287, 402; Brocato et al. 1998, MNRAS 295, 711). Indeed, at very low metallicities, the agreement between predicted and observed lower main-sequences (in globular clusters) is remarkably good (also see Baraffe et al. 1997, A\&A, 327, 1054). However, at moderately high metal abundances, all current models show appreciable discrepancies in the sense of being too hot/blue at faint $M_{V}$ 's, suggesting that there are still some missing sources of opacity (see, as well, Baraffe et al. 1998, A\&A, 337. 403). The Chabrier \& Baraffe study pointed out that significant departures from non-ideality occur only below the atmospheric layers, even in the densest stars near the $\mathrm{H}$-burning limit, and hence that errors arising from the use of a Saha-type equation of state throughout the atmosphere are negligible. They also noted that, near the bottom of the main sequence, the deuterium lifetime against proton capture is much less than the convective mixing timescale; consequently, instantaneous mixing cannot be assumed, and a deuterium gradient will develop in the cores of the lowest mass stars. Kroupa \& Tout discussed the considerable sensitivity to metallicity of the minimum in the derivative of mass with respect to $M_{V}$ that occurs on the lower main sequence (due mainly to the formation of $\mathrm{H}_{2}$ ). This minimum would correspond to a peak in the observed luminosity function of a chemically-homogeneous stellar population. Detection of that peak would offer important constraints on the properties of low-mass stars in the observed system.

\subsection{Brown Dwarfs and Extra-Solar Planets}

The extension of the stellar sequence into the brown dwarf regime is now well established with the discovery of many more substellar objects (those with masses below $\approx 0.075 \mathcal{M}_{\odot}$ ) (e.g., Stauffer et al. 1998, ApJ 499, L199; Martín et al. 1998, ApJ 509, L113; Kirkpatrick et al. 1999, ApJ 519, 802; Reid et al. 1999, ApJ 521, 613; and references therein). Indeed, Reid et al. conclude from their analysis that the available observations are consistent with a power-law mass function having an exponent $1<\alpha<2$ (similar to that determined for low mass stars in the solar neighborhood). However, the implied mass density is still well below that needed for brown dwarfs to be the main contributor to the Galaxy's dark matter.

Burrows et al. (1997, ApJ 491, 856) and Burrows \& Sharp (1999, ApJ 512, 843) have presented state-of-the-art models for brown dwarfs and extra-solar planets. Non-gray atmospheres, spectra, colors, and evolutionary sequences have been calculated for objects with masses ranging from 0.2 to $0.0003 \mathcal{M}_{\odot}$ and effective temperatures down to as low as 100 $\mathrm{K}$. Moreover, isochrones are provided for ages from $10^{6.5}$ to $10^{\mathbf{9 . 5}} \mathrm{yr}$, and both the interior structures and the surface layers are discussed in considerable detail. It is a testament to the complexity of the models reported by Burrows \& Sharp that the abundances of 330 gasphase species and 120 condensates are followed: the changing chemistry with temperature permits the definition of a sequence of spectral types for brown dwarfs. The theoretical study of such objects is truly well under way. 


\section{WHITE DWARFS}

Large scale surveys have greatly increased the number of known white dwarfs, both from ground-based observations (the EC survey: Stobie et al. 1997, MNRAS 287, 848; Kilkenny et al. 1997, MNRAS 287, 867; the HQS: Engels et al. 1998, A\&AS 128, 507) and from space (ROSAT: Marsh et al. 1997, MNRAS 286, 369; EUVE: Vennes et al. 1997, ApJ 480, 714). Follow-up spectroscopic observations are going on and produce atmospheric parameters (Homeier et al. 1998, A\&A 338, 563). The recently issued new version of the McCook and Sion's catalog $(1999$, ApJS 121,1) contains 2400 spectroscopically identified white dwarfs. Parallaxes issued from Hipparcos for the brightest white dwarfs and some white dwarfs components of binary systems have confirmed the Chandrasekhar's theoretical mass-radius relation (Shipman et al. 1997, ApJ 488, L43; Provencal et al. 1998, ApJ 494, 759; Vauclair et al. 1997, A\&A 325, 1055). Sirius B has been revisited and new EUVE data place it also on the expected mass-radius relation (Holberg et al. 1998, ApJ 497, 935).

The white dwarf cooling sequence may be compared to the white dwarf luminosity function (WDLF) to derive the age of the local galactic disk. This requires a better knowledge of the WDLF cool end which has been significantly improved (Legget et al. 1998, ApJ 497, 294; Garcia-Berro et al. 1999, MNRAS 302, 173). The cooling sequence has been observed in the closest globular clusters (Richer et al. 1997, ApJ 484, 741) where it will provide an independent age determination as soon as the coolest white dwarfs are detected. In the open cluster M67, the age derived from the white dwarf sequence agrees satisfactorily well with the one derived from the MS turn-off (Richer et al. 1998, ApJ 504, L91). It is suggested that a large fraction of the observed micro-lensing events could be due to a white dwarf galactic halo population (Graff et al. 1998, ApJ 499, 7; Chabrier 1999, A\&A 513, L103). These developments have prompted new generations of white dwarf evolutionary models, incorporating the best up-to-date physics (Benvenuto \& Althaus 1997, MNRAS 288, 1004; 1999, MNRAS 303, 30; Garcia-Berro et al. 1997, MNRAS 289, 973). Evolutionary models including new opacities (OPAL) and diffusion have also been used to study the carbon dredge up in He rich envelope white dwarfs. The derived $\mathrm{C} / \mathrm{He}$ distribution fits the observations for $\mathrm{He}$ mass layer in better agreement with standard evolution theory than previous works (MacDonald et al. 1998, MNRAS 296, 523). The angular momentum evolution has been investigated both from observations, searching for rotation velocity (Koester et al. 1998, A\&A 338, 612) and from theoretical studies: Spruit (1998, A\&A 333, 603) suggests that small non-axisymmetrical mass loss on the AGB may produce the 1 day rotation period frequently observed in white dwarfs. Observations of white dwarfs in the open cluster NGC 2516 lead to a revised upper mass limit of 5-6 $\mathrm{M}_{\odot}$ for white dwarf progenitors (Jeffries 1997, MNRAS 288, 585). The exceptional star $\mathrm{H} 1504+65$ has been revisited in the EUV: it is a hot post AGB entering the white dwarf sequence after complete loss of its outer layers and it shows only $\mathrm{O}$ and $\mathrm{Ne}$ with abundances in agreement with theoretical expectation for $3 \alpha$ processed matter (Werner \& Wolff 1999, A\&A 347, L9).

Progresses in atmosphere modeling include the introduction of Ly $\alpha$ and $\mathrm{Ly} \beta$ absorption lines profiles from Allard et al. $(1998, A \& A 335,1124)$ which led to a better determination of $T_{e}$ from far UV spectra (Koester et al. 1998, A\&A 336, 276). The observations in the UV (with ORPHEUS, EUVE) have provided a lot of new data on hot white dwarfs allowing to determine their photospheric metal abundances, to revise their effective temperature (Barstow et al. 1998, MNRAS 299, 520), to test the predictions of the radiative levitation theory and better describe the links with white dwarf progenitors (Barstow et al. 1997, MNRAS 286, 58; Vennes et al. 1998, ApJ 500, L41; Holberg et al. 1999, ApJ 517, 850). Some particular objects still escape complete understanding, as HZ43 which shows no metals at a $T_{e}$ where radiative levitation does predict some, may be indicating the efficiency of a competing mechanism like mass loss (Dupuis et al. 1998, ApJ 500, L45). Mass loss has been effectively detected in some hot pre-white dwarfs of the PG1159 type (Kruk \& Werner 1998, ApJ 502, 858; Koesterke \& Werner 1998, ApJ 500, L55; Koesterke et al. 1998, A\&A $330,1041)$ and could still be efficient in hot DAs. The hot DA G191-B2B is one of the best studied case: the opacity in the EUV is dominated by Fe (Wolff et al. 1998, A\&A 329, 
1045); stratified $\mathrm{H} / \mathrm{He}$ atmospheres with mass loss have been suggested to fit the UV spectra (Barstow \& Hubeny 1998, MNRAS 299, 379; Barstow et al. 1999, MNRAS 307, 884). More recently, a self consistent calculation of the model atmosphere including both gravitational settling and radiative levitation seems to give a good fit to the EUVE spectrum without a need for accretion of metal rich material or for mass loss (Dreizler \& Wolff 1999, A\&A 348, 189 ). Both cases, with and without mass loss, probably exist in nature. More theoretical work has been triggered by these observations, e.g. on the competition of diffusion with mass loss (Unglaub \& Bues 1998, A\&A 338,75). On the cool white dwarfs side, the current interests for extrasolar planets has triggered investigations on the consequence of planets and/or comets orbiting and ultimately falling onto white dwarfs, as a possible explanation for the metal abundances found in cool white dwarfs (Li et al. 1998, ApJ 503, L15; Parriot \& Alcock 1998, ApJ 501, 357; Zuckerman \& Reidl 1998, ApJ 505, L143). The need for better model atmosphere at low $T_{e}$, has led to new generations of $\mathrm{H}$ model atmospheres, including $\mathrm{H}_{2}$ (Saumon \& Jacobson 1999, ApJ 511, L107). This may be also pertinent for the problem of the non-DA gap in which additional opacity in He rich white dwarfs is invoked (Malo et al. 1999, ApJ 517, 901).

Asteroseismology is a powerful tool to test the internal structure and evolution of white dwarfs. If discovering new pulsating white dwarfs is rare, due to their faintness (Giovannini et al. 1998, A\&A 329, L13; Jordan et al. 1998, A\&A 330, 277), the observational asteroseismology has made some important breakthroughs. The PG1159 pulsators (or GW Vir) have been all observed via ground-based network. Among them, the case of PG0122+200 is unique in having its cooling rate dominated by neutrino losses, and is a potential neutrino laboratory (O'Brien et al. 1998, ApJ 495, 458). The GW Vir instability is now well defined from HST observations and blanketed NLTE model atmospheres and extends from $140000 \mathrm{~K}$ down to $80000 \mathrm{~K}$; in addition in having more $\mathrm{C}$ and $\mathrm{O}$ than their non pulsators twins, the pulsators show strong $\mathrm{N}$ in their spectra which may be the clue of the excitation mechanism (Dreizler \& Heber 1998, A\&A 334,618). The presence of $H$ in the outer layers is no longer poisoning the excitation mechanism, as demonstrated by the existence of the pulsating "hybrid" PG1159 star HS2324+3944 whose atmosphere contains $17 \%$ of $\mathrm{H}$ : about 20 pulsation frequencies have been detected in this still unique object (Silvotti et al. 1999, A\&A 342, 745). The DB instability strip is not so well explored from an asteroseismologic point of view. However, its theoretical boundaries have been considerably improved and it was shown that the blue edge is quite sensitive to the amount of $\mathrm{H}$ hidden in the He dominated atmosphere, a quantity still out of reach (Beauchamp et al. 1999, ApJ 516,887 ). The blue edge of the DBV instability strip has been used to test models for DB white dwarfs, including new theory for convection (Althaus \& Benvenuto 1997, MNRAS $288, \mathrm{L35}$ ). The above mentioned uncertainty on the $\mathrm{H}$ content and its consequence on the blue edge location does not yet give a satisfactory test of the underlying convection theory. Much works have been devoted to the DAV or ZZ Cetis. The role of the convection zone on the driving, has been explored and shown to be the dominant driving mechanism (Goldreich \& Wu 1999, ApJ 511, 904). Considerable interest has been devoted to the massive DAV BPM37093 which theoretical models predict to be partially crystallized in the core (Winget et al. 1997, ApJ 487, L191). New methods of mode identification are needed for these stars showing a too small number of modes: time resolved spectroscopy is now at reach with the new large telescopes; multi-multi site campaigns reveal to be necessary because of mode amplitude variations (Kleinman et al. 1998, ApJ 495, 424); simultaneous UV and optical observations exploit the amplitude difference predicted for various $\ell$ degree of the oscillations to identify $\ell$. Modeling DAV to fit the best available observational data has been relatively successful for G117-B15A and R548 (Bradley 1998, ApJS 116, 307). The best fit models seem to prefer $\mathrm{H}$ mass layer of the order of $10^{-4}$, but the question of the $\mathrm{H}$ outer mass in DA is still controversial. The presence of a brown dwarf around the peculiar IR excess DAV G29-38 is excluded on the basis of new IR speckle interferometry at KECK, the IR excess is probably due to a dust cloud (Kuchner et al. 1998, ApJ 508, L81). The potentiality of using asteroseismology of white dwarfs to derive the internal differential rotation has been analyzed. The inversion technique has limited application in this case 
because of the too small number of modes but interesting constraints on the differential rotation could be derived (Kawaler et al. 1999, ApJ 516, 349).

More magnetic white dwarfs have been discovered. Some have a field as strong as hundreds of MG (Burleigh et al. 1999, ApJ 510, L37; Schmidt et al. 1999, ApJ 512, 916; Ferrario et al. 1997, MNRAS 289, 105; and 1997, MNRAS 292, 205). Some others have more modest fields of a few MG (Moran et al. 1998, MNRAS 299, 218; Ferrario et al. 1998, MNRAS 299, L1). But a surprising result is that magnetic white dwarfs have larger mean mass than non magnetic ones, some being both massive and rapidly rotating, which is suggestive of a possible coalescence of the components of double degenerate systems. New He rich magnetic white dwarfs have been found in addition to the previous unique case GD229 (Reimers et al. 1998, A\&A 337, L13). The case of GD229 has been revisited and the long standing mysterious absorption feature is identified as He line in a strong magnetic field (Jordan et al. 1998, A\&A 336, L33; Jones et al. 1999, A\&A 343, L91).

White dwarf companions occur in interacting binary systems (novae, cataclysmic variables etc). Double degenerate systems received much attention as SNIa progenitors and as possible source of gravitational wave background. These subjects are out of the scope of this review, limited to single white dwarfs, and have to be found in the appropriate chapters.

\section{TRANSPORT PROCESSES IN STELLAR INTERIORS}

\subsection{Convection}

The treatment of convection remains one of the main weaknesses of stellar structure theory: there is still no simple, reliable way to fit an atmosphere model to convective layers beneath. Contrary to claims made a few years ago, all mixing-length prescriptions contain at least one adjustable parameter; and when this parameter is calibrated on the sun, there is no guarantee that its value can be applied to other stars. It actually depends on the location of the star in the HR diagram, as was shown by Ludwig et al. (1999, A\&A 346, 111), who determined through hydrodynamical simulations the relevant parameter of the two most popular prescriptions, namely the classical mixing-length recipe and the so-called "full turbulent spectrum" version of Canuto, Goldman and Mazzitelli (1996, ApJ 473, 550). The latter prescription is favored by some because the convective transport is less efficient in the subphotospheric layers than in the classical MLT; therefore it produces a temperature stratification which is in closer agreement with the helioseismic data (Antia \& Basu 1997, SCORe'96). For this reason it also permits to fit with the same parameter the interior model of the sun and the intensities of the Balmer lines (Bernkopf 1998, A\&A 332, 127). But when applied to the PMS evolution of solar-type stars, this FST predicts a very strong Li destruction which is incompatible with the observations (Ventura et al. 1998, A\&A 1011).

There is some hope to improve the situation by going beyond the mixing-length approach, and by using instead a non-local description involving higher order moments (cf. Xiong 1989, A\&A 209, 126; Gehmeyer \& Winkler 1992, A\&A 253, 92; Grossman 1996, MNRAS 279, 305; Canuto 1997, ApJ 482, 827). The set of equations derived by Canuto and Dubovikov (1998, ApJ 493, 834) has been tested against 3-dimensional simulations of shallow convection zones, and the results are encouraging (Kupka 1999, preprint astro$\mathrm{ph} / 9909331$ ), although they seem to underestimate the amount of convective penetration into the neighboring stable layers. These one-dimensional representations of turbulence improve also the modelization of stellar pulsations (Bono and collaborators, see $\S 5.2$; Yecko et al. 1998, A\&A 336, 553).

But with the ever increasing computer power, the future clearly belongs to the more realistic, 3-dimensional simulations. Those performed for a while already by Nordlund, Stein and their collaborators describe the solar photosphere with great accuracy; although they are limited to the uppermost layers of a star, they give some clues on what is occurring deeper down. For instance, they suggest that stellar convection is highly intermittent, with strong, long-lived downflows, and these are present in all simulations of stratified convection, as confirmed again by the most recent ones (Brummel et al. 1996, ApJ 473, 
494; Freytag et al. 1996, A\&A 313, 497; Stein \& Nordlund 1998, ApJ 499, 914; Rast 1998 , J. Fluid Mech. 369, 125). Such plumes have now been detected in the sun, through time-distance tomography (D'Silva et al. 1996; Duvall et al. 1997). Plumes have certainly a strong impact on the dynamics of stellar convection zones, which cannot be reduced to a turbulent diffusion, as in MLT; for a recent account on their properties, see Zahn 1999 (preprint astro-ph/9909288). Moreover, they probably play a key role in the dynamo mechanism, since they are able to "pump down" the magnetic field (Brandenburg et al. 1996, J. Fluid Mech. 306, 325; Tobias et al. 1998, ApJ 502, 177; Dorch 1998, Ph.D. thesis http://www.astro.ku.dk/ ${ }^{\text {dorch}) . ~}$

Convective penetration into the adjacent stable layers remains an unsolved problem, and its modelization requires yet another adjustable parameter. In numerical simulations convection penetrates much deeper than allowed by observed evolutionary tracks (for massive stars) or by helioseismology (for the sun). The main reason is that these simulations are not realistic enough, for lack of spatial resolution, and therefore their results must be rescaled to the actual conditions. Hence the first step should be to test numerically the scaling relations which have been proposed, by varying the relevant parameters, a strategy which has been adopted by Singh et al. (1998, A\&A 340, 178).

Numerical simulations also helped greatly to identify the excitation mechanism of acoustic waves in the sun (Nordlund \& Stein 1998, IAU Symp. 185; Georgobiani et al. 1999, BAAS 194,\# 56.05). Likewise they have been used to estimate the flux of internal gravity waves produced at the base of a convection zone (Kiraga et al. 1999, ASP Conf. Ser. 173, 269). The effect of rotation has been investigated in the so-called $f$-plane approximation by Brummel et al. (1998, ApJ 493, 955). An ambitious project by the same team aims at modeling the whole solar convection zone, in spherical geometry (Elliott et al. 1999, SOHO9 workshop); preliminary results reveal for the first time a significant departure from cylindrical rotation, as observed in the sun.

\subsection{Atomic diffusion}

Over the last three years the role of atomic diffusion in stellar evolution has been further confirmed by numerous studies of helioseismology (Brun et al. 1998, ApJ 506, 913; Fiorentini et al. 1999, A\&A 342, 492; Bahcall et al. 1997, PRL 78, 171; Guzik \& Swenson 1997, ApJ 491, 967; Morel et al. 1997, A\&A 327, 349 among others) showing the importance of He settling, some suggesting that the effect of the settling of metals is also important (Guenther \& Demarque 1997, ApJ 484, 937).

There are however two properties which need to be included in self consistent atomic diffusion calculations which were not included in those stellar evolution studies: first, radiative accelerations, and second, the detailed variations of Rosseland averaged opacities as chemical abundances change as a consequence of atomic diffusion and nuclear reactions. Until recently it was impossible to properly include these effects in stellar evolution codes since the atomic data needed to calculate radiative accelerations were not available nor were the detailed frequency-dependent contribution of each species to the opacity. However the availability of the OPAL and Opacity Project data have now made possible completely self consistent stellar evolution calculations taking atomic diffusion into account. Using these data Richer et al. (1998, ApJ 492, 833.) have described how radiative accelerations and Rosseland opacities could be calculated during stellar evolution and have shown the importance of recalculating them at every time step of the stellar evolution as the abundances of the species included in OPAL tables (Rogers \& Iglesias 1992, ApJS 79, 507) change. They also analyzed the effect of correcting for the momentum given to electrons in a photoionization using Opacity Project data (Seaton et al. 1994, MNRAS 266, 805).

Following this approach, Turcotte et al. (1998, ApJ 504, 539) have analyzed in detail the effect of atomic diffusion on solar models. They have shown that radiative accelerations were not negligible. For certain Fe peak elements they can reach $40 \%$ of gravity at the bottom of the solar convection zone. They alter the Rosseland opacity by up to $0.5 \%$ and reduce the neutrino flux by $3 \%$. Atomic diffusion leads to a change of up to $8 \%$ 
in the Rosseland opacity. Turcotte et al. show how a careful use of tabulated radiative accelerations and tabulated opacity tables in solar evolution codes can lead to a residual of only $0.1 \%$ with respect to the complete solution obtained with monochromatic opacities.

Using the mixing length calibrated with solar models, Turcotte et al. (1998, ApJ 504, 559) calculated evolutionary models for stars of 1.1 to 1.5 solar mass without any arbitrary parameter and allowing for the diffusion of 28 species. They showed that iron surface overabundances reach factors of 3-6 and cause an increase by a factor of up to 5 in the mass of the convection zone. The abundance anomalies resemble those of Fm stars but are larger than observed, suggesting that some perturbing hydrodynamical process reduces the anomalies. The Fe overabundances and CNO underabundances they obtain in F stars are not observed in all $\mathrm{F}$ stars of the $\mathrm{Li}$ gap. This suggests that atomic diffusion is not the only process responsible for the $\mathrm{Li}$ gap in open clusters.

Richer et al. (2000 ApJ, in press) extended those calculations to Am stars (1.45 to 3.0 solar mass). These models develop iron peak convection zones centered at a temperature of approximately $200000 \mathrm{Kelvin}$. These authors then assume that there is sufficient overshoot to homogenize the surface regions between the hydrogen, helium and iron peak convection zones, and show that the surface abundance variations that are produced, without any arbitrary parameter, closely resemble the abundance anomalies of AmFm stars except that they are larger by a factor of about 3 .

They added turbulent diffusion in the outer stellar regions in order to improve the agreement with the observed anomalies in AmFm stars. They found that so long as turbulence was strong enough to mix approximately the outer $10^{-5}$ of the stellar mass, the observed abundance anomalies of AmFm stars were reasonably reproduced by models of the same age as the stars. They did detailed comparisons for Sirius A as well as five stars of open clusters. For instance in Sirius A, abundances were observed for 16 elements. Twelve are well reproduced, three are not so well reproduced and one is a very uncertain observation.

Such calculations could in principle be extended to HgMn stars. These have been very carefully observed recently, in particular by Leckrone et al. $(1999$, AJ 117, 1454) and Woolf \& Lambert (1999, ApJ 521, 414). However, in those objects the very large isotopic abundance anomalies of elements such as $\mathrm{Hg}$ and $\mathrm{Pt}$ can most probably be reproduced only by separation in the atmosphere (Proffitt et al. 1999, ApJ 512, 942) and calculations such as those mentioned above for AmFm are not currently possible there. Spectral lines are narrower in the atmosphere than deeper in the star, and the detailed calculation of the effect of blends requires an atomic data bank whose energy levels are known more accurately than in OPAL or the Opacity Project, as well as a finer grid than used by OPAL. Seaton (1999 MNRAS, in press) has applied Opacity Project radiative accelerations to investigative studies of $\mathrm{HgMn}$ star models. While he did not calculate evolutionary models for those stars, his results suggest that atomic diffusion in these objects should lead to the appearance of iron peak convection zones.

The coupling of large atomic data banks to stellar evolution codes opens a new window for stellar evolution. It becomes possible to determine quantitatively the importance of stellar hydrodynamic processes through their effect on the surface abundances. The use of large telescopes and space telescopes makes it possible to determine the abundances of most chemical species. Their determination in cluster AmFm and HgMn stars would lead to a very well defined challenge for stellar evolution. Atomic data banks also need to be extended to include species heavier than nickel since many are observed in AmFm and HgMn stars; their frequency coverage as well as the accuracy of the energy determinations also need to be improved so that the effect of blends may be determined even in atmospheric regions. These are major challenges but recent progress suggests that they are worth accepting. 


\subsection{Rotational mixing}

In recent years more evidence has been gathered that some mixing must occur in the radiative interior of stars, and there are some indications that such mixing is related to rotation. This is attested by the overabundance of $\mathrm{He}$ in rapidly rotating massive stars (Herrero et al. 1999, A\&A 348, 542), and by the fact that, in solar-type stars, fast rotators as a rule suffer less Li depletion than slow rotators (cf. Balachandran et al. 1998, ASP Conf. Ser. 154, 111; Randich et al. 1998, A\&A 333, 591). The current interpretation invokes as main physical mechanism the so-called rotational mixing: it consists of the classical, thermally driven meridional circulation, associated with shear instabilities which are generated by the differential rotation (see Pinsonneault's comprehensive review in ARA\&A 35, 557; cf. also Chaboyer 1998, IAU Symp. 185). Recent improvements of the theory have dealt with the effect of the composition gradient, both on the circulation (Maeder \& Zahn 1998, A\&A 334,1000 ) and on the stratified shear instability (Maeder 1997, A\&A 321, 134; Talon \& Zahn 1997, A\&A 317, 749). Non standard models of rotating massive stars have been built which include this rotational mixing, and they agree reasonably well with the observations (Talon et al. 1997, A\&A 322, 209; Denissenkov et al. 1999, A\&A 341, 181; Langer et al. 1999, A\&A 346, 37; Meynet \& Maeder 2000, to appear in A\&A). Similar calculations have been carried out for intermediate-mass stars, on the hot side of the Li gap, and they too yield the observed $\mathrm{LiBeB}$ abundances on the main sequence (Talon \& Charbonnel 1998, A\&A 335, 959) and beyond (Charbonnel \& Talon 1999, to appear in A\&A).

The situation is less satisfactory for solar-type stars. The theoretical prediction that the amount of mixing is correlated with the loss of angular momentum accounts fairly well for the observations of $\mathrm{Li}$ depletion; for instance it explains why tidally locked binaries preserve more Li than single stars, as observed in the Hyades. However rotational mixing alone is unable to render nearly uniform the internal rotation of the Sun, as inferred from helioseismology, which means that some other mechanism must contribute to the transport of angular momentum. Moreover, such deep rotational mixing would cause some depletion of $\mathrm{Be}$, whose underabundance in the solar atmosphere has been seriously questioned by Balachandran and Bell (1997, Bull. AAS 191, \#74.08). Two other transport processes have been considered, which may enforce this uniform rotation in the sun: low frequency internal gravity waves produced at the base of the convection zone (Kumar \& Quataert 1997, ApJ 475, L133; Zahn et al. 1997, A\&A 322, 320; Kumar et al. 1999, to appear in ApJ) and magnetic torquing due to a primeval field (Gough \& McIntyre 1998, Nature 394, 755; Barnes et al. 1999, ApJ 511, 466), but far more work is needed to settle the question.

Another possibility is that the mixing in solar-type stars is confined just in the tachocline, this thin layer where the rotation profile changes abruptly from uniform in the radiative interior to differential, i.e. latitude dependent, in the convection zone. Based on the purely hydrodynamical description of the tachocline given by Spiegel and Zahn (1992, A\&A 265, 106), the depletion of LiBeB during the spin-down of the sun has been calculated by Brun et al. (1999, to appear in ApJ), and their results agree rather well with the observed values. But the structure of that layer, whether it is turbulent, what then causes the turbulence, and which role the magnetic field plays, all these issues are still matter of debate (cf. Gough \& McIntyre 1998, Nature 394, 755; Charbonneau et al. 1999, to appear in ApJ).

\section{HELIO- AND ASTEROSEISMOLOGY}

Testing models of the sun's interior used to be one of the main applications of helioseismology. To a large extent this application was already exploited. The bottom line is that results of seismic sounding support the standard scenario of solar evolution. This result contributed to the view, which is now prevailing, that the inadequacies in models of the sun's interior structure cannot be regarded as a viable solution of the neutrino deficit problem. The differences in structural parameters between the sun and its models revealed by frequency inversions are small and although statistically significant (e.g. Brun et al. 1998, ApJ 306, 913) are not challenging the standard model. The differences in the radiative 
interior may be accounted for by modifications of the opacity within its uncertainty (Tripathy \& Christensen-Dalsgaard 1998, A\&A 337,679) and those in the outer convective zone by means of acceptable modifications in treatment of convection (Rosenthal et al. 1998, SOHO6/GONG98, 521).

Recent highlights in helioseismology concern primarily internal flows in the sun. The overall picture of the internal rotation established in late 1980's has not been challenged by recent helioseismic inversions but new and important details were found. Furthermore, we obtained first data on other types of large scale flows in the sun's interior.

Schou et al. (1998, ApJ 505, 390) produced a new two-dimensional map of the rotation rate for the convective zone and outer parts of the radiative interior, based on the SOHO/MDI data, with sharper details about the tachocline - the zone where the rotation changes abruptly from latitude dependent to uniform. Probing this zone is essential for understanding angular momentum transport from the radiative interior to the convective envelope as well as for theories of magnetic field generation. Next important detail concern the initial growth of the rotation rate down from photosphere. This provides a clue to understanding differences in rotation of various photospheric tracers. Finally, a significant departure from the usual rotation law - described by a second order polynomial in $\cos ^{2} \theta$ - was found at latitudes above $70^{\circ}$. Earlier, the same inference based only on f-mode data was made by Kosovichev and Schou (1997, ApJ 482, L207).

Properties of the tachocline, such as its extent and shape, were studied also by Basu and Antia (1998, SOHO6/GONG98, 705) who used data from the GONG network and Charbonneau et al. (1999), who used the data from LOWL instrument and by Larsen et al. (1999, 9th SOHO Workshop, in press), who used MDI, GONG and LOWL data sets combined. Physics of the tachocline was investigated by Gough and McIntyre (1998, Nature $394,755)$.

Duvall et al. (1997, Solar Phys. 170, 3) determined the vertical structure of supergranular velocity field through analysis of the sound travel data from the SOHO/MDI instrument. The inferred depth of the supergranular layer was $8 \mathrm{Mm}$. A similar analysis lead also to detection of the subsurface poleward meridional circulation with velocities $\sim 20 \mathrm{~m} / \mathrm{s}$ (Giles et al. 1997, Nature 390, 52). Significant velocity measurements were made down to depth of $26 \mathrm{Mm}$. Subsequent measurements of the circulation velocities were made with the ring diagram analysis by Basu et al. (1998, SOHO6/GONG98, 705) and by González Hernández et al. (1998, SOHO6/GONG98, 781). Data on meridional circulation are important for theories of rotation in stellar convective envelopes.

In contrast to helioseismology, which is an accomplished field of research, asteroseismology is still in its infancy phase. It has been most successful in the case of oscillating white dwarfs. Recent results for these stars are mentioned in $\S 6$ of this report.

Among main sequence variable stars the multimode pulsation is common. Thus, there are many objects of interest for asteroseismic analyses. The ultimate goal is to construct models of the objects which reproduce measured frequencies; it has not been yet fully achieved. Subject of intense observational and theoretical investigations were two $\delta$ Scuti stars FG Vir (Breger et al. 1998, A\&A 331, 271; Guzik et al. , 1998, PASP Conf. Ser. 135, 470; Viskum et al. 1998, A\&A 335, 449; Breger et al. 1999, A\&A 341, 151) and XX Pyx (Handler et al. 1997, MNRAS 386, 303; Pamyatnykh et al. 1998, A\&A 333, 141). Dziembowski and Jerzykiewicz (1999, A\&A 341, 480) modeled the multimode $\beta$ Cephei star DD Lac. Matthews et al. (1999, ApJ 511, 422) used the p-mode frequency spacings of 12 roAp stars to infer the radii and with data on $T_{\text {eff }}$ determined the luminosities, which they compared with the luminosities derived from the Hipparcos parallaxes.

Multimode pulsation has been recently discovered in a number of B-type subdwarfs (Kilkenny et al. , 1997, MNRAS 285, 640; Koen et al. , 1997, MNRAS 285, 645; Stobie et al. 1997, MNRAS 285, 651). For one of these stars (PG 1605) Kawaler (1999, PASP Conf. Ser. 169,158$)$ constructed an evolutionary stellar model which yields an approximate fit to frequencies of the dominant peaks in the amplitude spectrum. 
Asteroseismology from space is just starting. Buzasi et al. (1999, to appear in ApJ) identified several normal mode frequencies in the giant star $\alpha$ UMa using data from the small telescope on the WIRE space mission. Three dedicated asteroseismic missions (COROT, MOST and MONS) are in the preparation phase.

Jean-Paul Zahn President of the Commission 